\title{
Plasma Erythropoiesis Stimulating Factor(s) in Neonatal Mice: In Vitro Dose Response and Chromatography Studies
}

\author{
TRULS SANENGEN AND SVERRE HALVORSEN
}

Departments of Pediatrics and Pathologv, Ullevål Hospital, Oslo, Norway

\begin{abstract}
High levels of plasma erythropoiesis stimulating factor(s) (ESF) have been found in neonatal WLOmice during rapid growth. A previous study on hypertransfused neonatal animals indicated that the high ESF could not be due to the concomitant postnatal anemia alone. The present investigation was performed to answer the question: Is the high plasma ESF in neonatal WLO-mice erythropoietin (Ep) alone, or Ep in combination with other factors? The ESF of plasma from 20-day-old animals and standard Ep were compared in a cell culture assay for ESF based on erythroid colony formation, and also by means of gel filtration chromatography and affinity chromatography. Nonfractionated plasma and standard Ep showed parallel dose response curves and additive activity in the ESF assay. After gel filtration the detectable ESF of plasma was eluted in the same position as that of standard Ep, corresponding to an estimated molecular weight range of 34-65000 daltons. The ESF of intact plasma, fractionated plasma, and standard Ep were identically bound to and eluted from the affinity chromatography column. These results show that the ESF of plasma from 20-day-old animals can neither be separated into several factors, nor distinguished from that of standard Ep by the methods used. It is therefore concluded that the high plasma ESF found in neonatal WLOmice probably consists of Ep alone. (Pediatr Res 21: 148151, 1987)
\end{abstract}

$\quad$ Abbreviations
CFU $_{\text {L, erythroid colony forming cell }}$
Ep, erythropoietin
ESF, erythropoiesis stimulating factor(s)
MW, molecular weight
PBS, phosphate-buffered saline
S-200 column, Sephacryl S-200 superfine column
WG-column, wheat germ lectin-Sepharose 6MB column
Vo, void volume
IGF-I, insulin-like growth factor I

Erythropoiesis in the adult mammal is regulated mainly through $\mathrm{Ep}$ in response to tissue hypoxia, although non-Ep factors may be involved (1). Erythroid progenitor cells in culture are also sensitive to these factors. Thus, cell culture assays for Ep based on erythroid colony formation determine ESF, which is the net activity of Ep and other erythropoietic stimulatory factors and possible inhibitory factors present in the samples.

Received November 7, 1985: accepted September 18, 1986.

Correspondence and reprint requests Dr. Truls Sanengen, 1460 Department of Pediatrics. Ullevål Hospital, 0407 Oslo 4, Norway.

Supported by the Norwegian Society for Fighting Cancer. The authors are grateful to Kirsten M. Østbye for excellent technical assistance
High levels of plasma ESF have been found in neonatal WLOmice during rapid growth with only a moderate concomitant postnatal anemia. The highest levels were found at about 20 days of age (2). Hypertransfusion reduced but did not totally suppress the ESF in 20-day-old animals (3). This indicated that the high levels found were not due to the anemia alone, and supported other studies indicating that regulation of erythropoiesis in the neonate differs from the adult.

Based on these findings $(2,3)$, the present investigation was performed to answer the question: Is the high plasma ESF found in neonatal WLO-mice Ep alone or Ep in combination with other factors? To accomplish this the ESF of plasma from 20day-old animals and standard Ep were compared in a cell culture assay and also by means of gel filtration chromatography and affinity chromatography.

\section{MATERIALS AND METHODS}

WLO-mice (4) of both sexes were sacrificed when 20 days old. The litter size was between five to 10 pups. Blood was collected in heparinized tubes (Caraway, American Dade, Division of American Hospital Supply Corporation, Miami, FL). The tubes were immediately centrifuged, and the plasma from each litter pooled and stored at $-70^{\circ} \mathrm{C}$. Plasma from several litters were pooled to achieve enough material for the various experiments. The plasma batches to be used for dose response studies in the ESF assay were dialysed for 3 days against distilled water (5). Precipitates were removed by centrifugation and the supernatants used in the assay. Undialysed plasma was used for the chromatography studies, and none of the chromatography fractions was dialysed before being assayed for ESF.

Sheep plasma erythropoietin (step III, Connaught Laboratories, Ontario, Canada) served as standard Ep. The lyophilized step III preparation was dissolved in $\alpha$-medium (Flow Laboratories, Herts, U.K.) and stored at $-70^{\circ} \mathrm{C}$.

ESF was determined by a modification of the cell culture assay described by Hågå and Falkanger (6), using hepatic $\mathrm{CFU}_{\mathrm{E}} \mathrm{S}$ from newborn WLO-mice (5). The test samples were added at a concentration of $100 \mu \mathrm{l} / \mathrm{ml}$, except for the dose response studies. $\alpha$-medium was added to a final volume of $250 \mu$ l per culture well. The number of erythroid colonies formed is proportional to the ESF present in the cultures in a log-dose fashion. The results were normalized to adjust for interassay variations (5).

Two dose response studies were carried out with the ESF assay. First, plasma from 20-day-old mice and standard Ep were tested separately in concentrations from 6.25 to $200 \mu \mathrm{l} / \mathrm{ml}$ and 6.25 to $200 \mathrm{mU} / \mathrm{ml}$, respectively. This was done to test parallelity of the two dose response curves. Second, the combined activity of plasma and standard Ep was studied. A fixed amount of plasma $(100 \mu \mathrm{l} / \mathrm{ml})$ was added to the cultures along with increasing concentrations of standard Ep (6.25-200 mU/ml). Parallel cultures with standard Ep alone were done in the same experiment. 
MW estimations were done by gel filtration chromatography at $4^{\circ} \mathrm{C}$ on a S-200 column (Pharmacia Fine Chemicals AB, Uppsala, Sweden). The column dimensions were 74 by $1.5 \mathrm{~cm}$, i.e. $130.8 \mathrm{ml}$ bed volume. The gel was equilibrated and the elutions carried out with PBS, $\mathrm{pH}$ 7.4. Flow was adjusted to 12 $\mathrm{ml} / \mathrm{h}$ and $1.2 \mathrm{ml}$ fractions were collected. The void volume $\left(\mathrm{V}_{\mathrm{o}}\right)$ was $45.6 \mathrm{ml}$. The samples applied were $2.5 \mathrm{ml}$ of plasma and standard Ep (625 mU), respectively: Each fraction was assayed for ESF. Protein standards and Blue Dextran 2000 (Gel Filtration Calibration Kit, Pharmacia Fine Chemicals AB) were dissolved in PBS. The plasma fractions that showed activity in the ESF assay were studied by affinity chromatography.

Affinity chromatography was carried out at $4^{\circ} \mathrm{C}$ on a WGcolumn (Pharmacia Fine Chemicals $\mathrm{AB}$ ). This column selectively binds glycoproteins containing certain sugar residues. A modification of the method of Spivak et al. was employed $(7,8)$. The column dimensions were 4.7 by $0.7 \mathrm{~cm}$, i.e. $1.8 \mathrm{ml}$ bed volume. The gel was equilibrated with PBS, $\mathrm{pH} 7.4$. Flow was adjusted to $12 \mathrm{ml} / \mathrm{h}$ and $1 \mathrm{ml}$ fractions were collected. Following application of the sample, $0.9 \mathrm{ml}$, the column was washed with PBS until the effluent was free of protein. Thereafter $1.8 \mathrm{ml}$ of a $1 \times$ $10^{-3} \mathrm{M}$ solution of $\mathrm{N}^{\prime} \mathrm{N}^{\prime}$-diacetylchitobiose (Sigma Chemical Company, St. Louis, MO) in PBS was applied. When the elution sugar had entered the gel bed flow was stopped and the column warmed by hand for $10 \mathrm{~min}$. Elution was then reassumed with PBS until the eluate was free of protein. The samples applied were $0.9 \mathrm{ml}$ of plasma, plasma fractions from the $\mathrm{S}-200$ column that had shown activity in the ESF assay (fractions 55, 57-62, 63-64) and standard Ep (225 mU). The S-200 column plasma fractions $57-62$ and $63-64$ had been pooled before studied by affinity chromatography, respectively. Each WG-column fraction was assayed for ESF. The gel was regenerated with $18 \mathrm{ml}$ of $1 \times 10^{-1} \mathrm{M}$ Tris/ $\mathrm{HCl}$ buffer containing $5 \times 10^{-1} \mathrm{M} \mathrm{NaCl}, \mathrm{pH}$ 8.5 , and $18 \mathrm{ml}$ sodium acetate buffer containing $5 \times 10^{-1} \mathrm{M}$ $\mathrm{NaCl}, \mathrm{pH}$ 4.5. The gel was then reequilibrated with PBS, $\mathrm{pH}$ 7.4.

A peristaltic pump (P-1, Pharmacia Fine Chemicals AB) and a fraction collector (Ultrorack 7000, LKB, Bromma, Sweden) were used for all the chromatographies. Protein contents of column effluents were continuously measured sphectrophotometrically at $280 \mathrm{~nm}$ (Uvicord 8003, LKB). All fractions were stored at $-30^{\circ} \mathrm{C}$ before being assayed for ESF. Column effluents free of protein and nonfractionated aliquots of the actual samples served as additional controls in these ESF assays.

The level of significance of differences between means were determined by Student's $t$ test.

\section{RESULTS}

In the first dose response study $25 \mu \mathrm{l} / \mathrm{ml}$ of plasma showed the same ESF activity as $6.25 \mathrm{mU} / \mathrm{ml}$ standard $\operatorname{Ep}(p>0.5)$, and $100 \mu \mathrm{l} / \mathrm{ml}$ of plasma equalled that of $25 \mathrm{mU} / \mathrm{ml} \mathrm{Ep}(p>0.5)$ (Fig. 1). Within this range the ESF response was proportional to the log of the dose of plasma or standard Ep added, with no difference in slope. Thus, within the given range of activity the two dose response curves were parallel. Plasma in a concentration of $200 \mu \mathrm{l} / \mathrm{ml}$ showed no activity in the ESF assay and was even inhibitory as compared to nonstimulated control cultures $(p<$ 0.01 ). This may reflect that plasma contained inhibitory factors and/or had replaced too much of the nutritional factors present in $\alpha$-medium.

Compared to plasma alone, the observed ESF response increased when increasing doses of standard Ep were added to the cultures along with the fixed amount of plasma $(100 \mu \mathrm{l} / \mathrm{ml}$ ) (Fig. $2)$. The highest combined response was found at a standard Ep concentration of $100 \mathrm{mU} / \mathrm{ml}(p<0.02)$. However, the maximal combined effect on erythroid colony formation equalled that observed when the culture system was maximally stimulated by standard Ep alone $(p>0.05)$. Thus, the effect of combining plasma and standard Ep was neither synergistic nor inhibitory, but truly additive in the present ESF assay.
The plasma fractions 57-62 from the S-200 column, corresponding to an estimated MW of 39-55000 daltons, showed high ESF levels as compared to column effluent free of protein $(p<$ 0.001 ) (Fig. 3). Much lower levels were found in the plasma

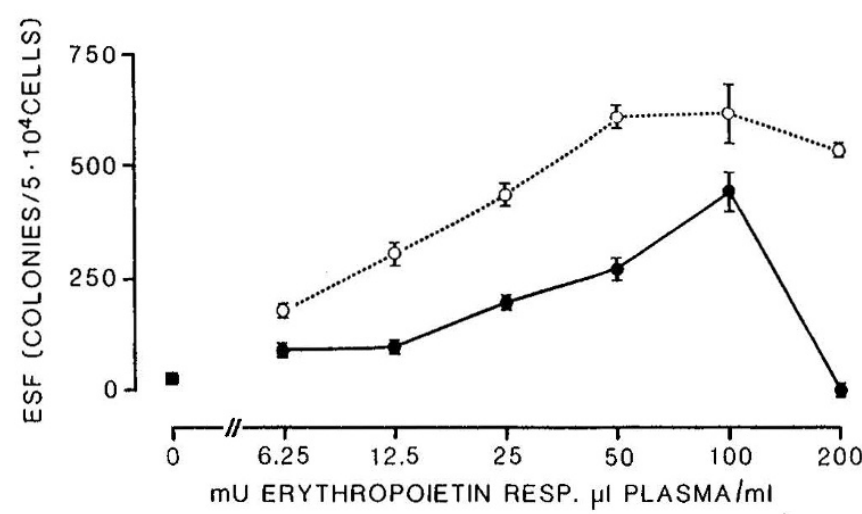

Fig. 1. Dose response curves for neonatal mice plasma and standard $\mathrm{Ep}$ in the ESF assay. ESF (colonies $/ 5 \times 10^{4}$ cells) found in increasing doses of standard $\mathrm{Ep}(\mathrm{O})(n=8)$ and plasma from 20-day-old WLOmice $(\Theta)(n=8)$, and in control cultures with no sample or standard Ep added $(\square)(n=16)$. The data are expressed as means \pm SEM.

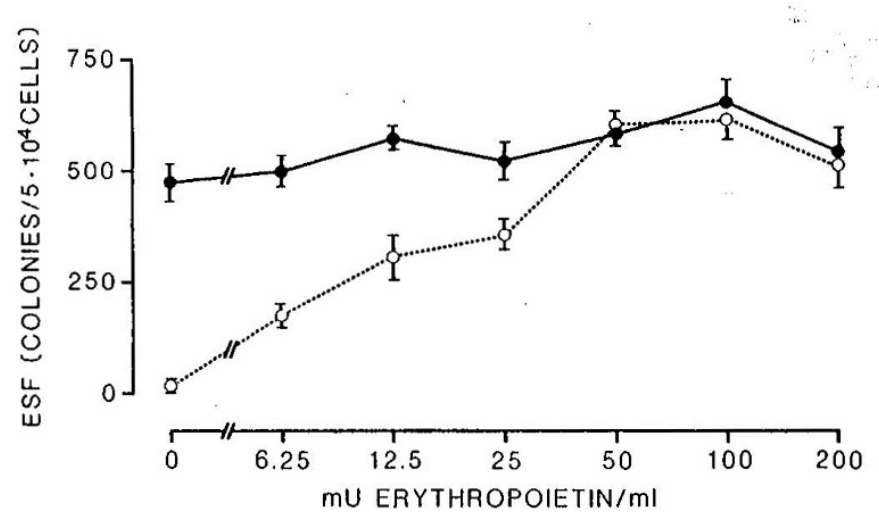

Fig. 2. Effect of adding increasing doses of standard Ep to a fixed amount of neonatal mice plasma in the ESF assay. ESF (colonies/5 $\times$ $10^{4}$ cells) found in $100 \mu \mathrm{l} / \mathrm{ml}$ of plasma from 20 -day-old WLO-mice with increasing doses of standard Ep added $(\Theta)(n=7-8)$ and in standard Ep alone $(O)(n=7-15)$. The data are expressed as means \pm SEM.

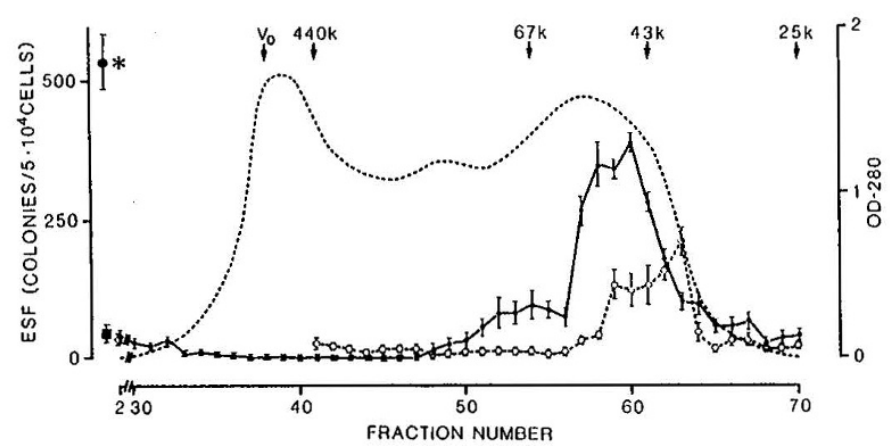

Fig. 3. Results of gel filtration chromatographies. ESF (colonies $/ 5 \times$ $10^{4}$ cells) $(0)(n=8)$ and OD-280 (--) found in the S-200 column fractions of plasma from 20-day-old WLO-mice. ESF found in nonfractionated plasma $\left({ }^{*}\right)(n=8)$, in the S-200 column fractions of standard $\mathrm{Ep}(\mathrm{O})(n=4)$ and cultures with no sample or standard Ep added $(\square)(n$ $=16)$. The data are expressed as means \pm SEM. The arrows indicate the elution positions of the protein standards (ferritin: $440 \mathrm{k}$, albumin: $67 \mathrm{k}$, ovalbumin: $43 \mathrm{k}$, chymotrypsinogen A: $25 \mathrm{k}$ ) and Blue Dextran $2000\left(\mathrm{~V}_{\mathrm{o}}\right)$. 
fractions 55,63 , and $64(p<0.025)$, while the fractions 33-48 were significantly inhibitory $(p<0.05)$. The ESF of standard Ep was eluted in fractions $59-63(p<0.05)$, corresponding to a MW of 37-48000 daltons. The fractions 44, 48-56 of the standard Ep were clearly inhibitory $(p<0.05)$. The S-200 column fractions 55-64 corresponded to a MW range of $34-65,000$ daltons.

The ESF of standard Ep, plasma, and the S-200 plasma fractions 55 and 57-62 were completely bound to the WGcolumn. There was no ESF to be found in the nonretarded fractions $(p>0.05)$. The ESF was recovered within the range of fractions 7-14 following application of the elution sugar (standard Ep: $8-14$, plasma: $7-13$, plasma fraction 55: 8, plasma fractions 57-62: 8-12) $(p<0.05)$ (Fig. 4). Data from the pooled plasma fractions 63-64 are not shown on Figure 4. The nonfractionated aliquot of this sample did not show activity in the ESF assay $(p>0.05)$, nor was there any ESF to be found in the nonretarded fractions from the WG-column $(p>0.05)$. Nevertheless, the fractions number 10 and 13 following application of the elution sugar showed a low ESF level $(p<0.05)$.

The S-200 and WG-column effluents free of protein did not have any inhibitory or stimulatory effect in the ESF assay as compared to control cultures with no sample or standard Ep added $(p>0.4)$, nor did they change the activity of $50 \mathrm{mU} / \mathrm{ml}$ standard $\operatorname{Ep}(p>0.5)$.

\section{DISCUSSION}

Several non-Ep humoral factors stimulate erythropoiesis in vivo, but their possible physiological role in the regulation of erythropoiesis remains unclear. Cell culture assays for Ep are also sensitive to such factors $(1,8-13)$. The observed effect varies with the culture system used, indicating that these factors may influence erythropoiesis in different ways and at various stages of differentiation $(8,14)$.

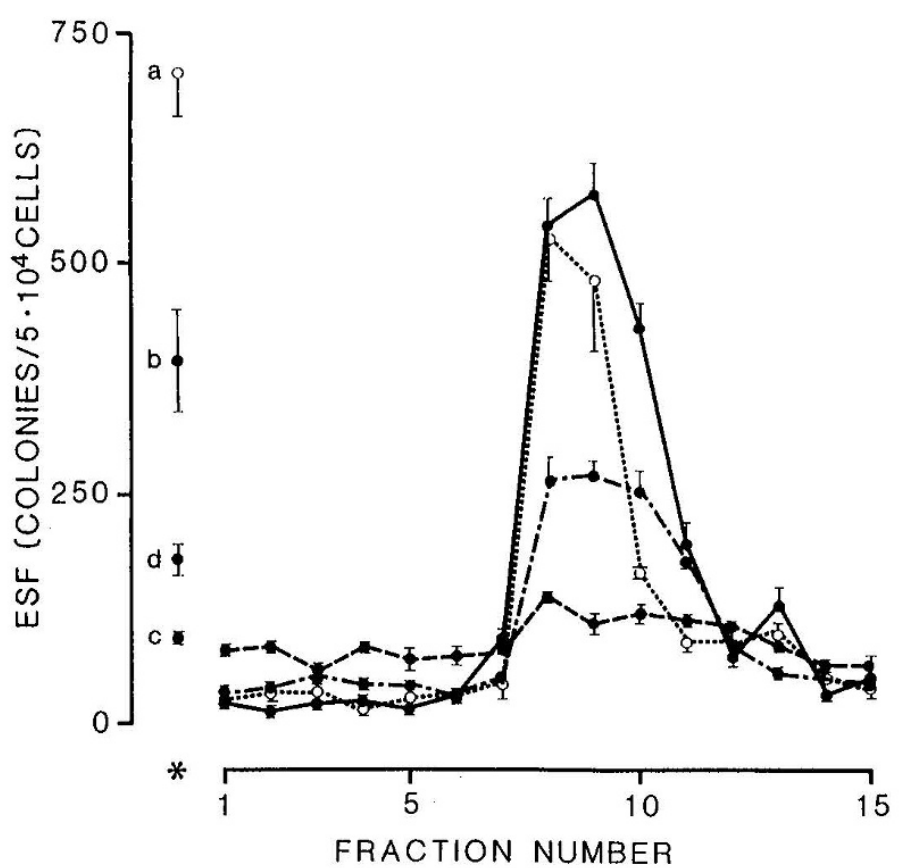

Fig. 4. Results of affinity chromatographies. ESF (colonies $/ 5 \times 10^{4}$ cells) found in the non fractionated aliquots $(*)$ and the WG-column fractions of standard $\operatorname{Ep}(a, O)$, plasma from 20-day-old WLO-mice $(b$, $\longrightarrow$ ) and the plasma fractions $55(c,-\infty)$ and 57-62 (d, -) from the S-200 column. Fraction 1 represents the first fraction collected after application of the elution sugar. The data are expressed as means $\pm \operatorname{SEM}(n=4)$.
Plasma from 20-day-old WLO-mice and standard Ep showed parallel dose response curves and additive activity in the cell culture assay for ESF based on erythroid colony formation (Figs. 1 and 2). These results indicate that the ESF in neonatal mouse plasma and standard Ep, respectively, have the same final effect on the target cell, the $\mathrm{CFU} \mathrm{E}_{\mathrm{E}}$. However, they do not prove that the effector mechanism(s) is identical.

After gel filtration most of the detectable ESF of plasma was eluted in the same position as that of standard Ep (Fig. 3). This shows that at least a significant part of the high plasma ESF is the result of one or more factors with a MW within the same range as Ep. IGF-I is a possible non-Ep factor. This somatomedin in physiological concentration stimulates the growth of murine $\mathrm{CFU}_{\mathrm{E}} \mathrm{S}$ in vitro (12). The somatomedins all have MWs of 610000 daltons. However, in the human serum they are bound to carrier proteins, and some of the circulating IGF-I has been shown to have a MW of about $38000 \pm 7000$ daltons at neutral $\mathrm{pH}(15)$. This is probably also the case in the mouse (16).

A plasma volume of $2.5 \mathrm{ml}$ was applied to the S-200 column, but the detectable ESF was eluted in a total volume of $10.8 \mathrm{ml}$ (nine fractions). Thus, the ESF of plasma was necessarily diluted by the gel filtration procedure. Having this in mind, the plasma fractions 57-61 showed approximately three times higher ESF levels than expected as compared to nonfractionated plasma (Fig. 3). Together with the observation that some plasma fractions were inhibitory in the ESF assay, this probably reflects that the S-200 column had separated some inhibitory factor(s) from the stimulatory activity. However, the data do not exclude the possibility that erythropoietic stimulatory factors of other MWs are present but not detected, because they are too much diluted and/or eluted along with inhibitory activity.

Affinity chromatography performed as described in "Materials and methods" is an accepted method to separate Ep from other non-Ep erythropoietic stimulatory factors $(7,8,17)$. In the present study the ESF of intact plasma and the ESF of plasma fractions from S-200 column were bound to and eluted from the WG-column as that of standard Ep (Fig. 4). This shows that a significant part of the plasma ESF is due to one or more glycoproteins containing at least one of the same sugar residues as Ep. Samples of $0.9 \mathrm{ml}$ were applied to the WG-column, but the detectable ESF was eluted in volumes varying from 1 to $7 \mathrm{ml}$. Therefore, the affinity chromatography procedure must also have diluted the ESF of the samples. However, some fractions showed remarkably high ESF levels as compared to the intact samples (Fig. 4). The WG-column fractions 8-10 of intact plasma showed approximately 10 times higher levels and those of the S-200 plasma fractions 57-62 were about seven times more active in the ESF assay than expected. It should be noted that all these fractions showed higher ESF levels than the intact samples. Even fractions 8-9 of standard Ep showed approximately four times higher ESF levels than one would expect from the dilution. This indicates that the WG-column had separated some inhibitory factor(s) from the stimulatory activity, both in plasma and standard Ep. Again, it is still possible that other erythropoietic stimulatory factors are not detected because they are too much diluted and/or eluted along with inhibitory activity.

The presented data show that the plasma ESF in 20-day-old WLO-mice cannot be separated into several factors by the methods used. Furthermore, the plasma ESF showed the same final biological activity on the $C F U_{E}$, was found within the same MW range and had at least one of the same sugar residues as the ESF of standard Ep. It is therefore concluded that the high plasma ESF found in neonatal WLO-mice, as represented by 20-day-old animals, probably consists of Ep alone.

The physiological stimulus behind the high Ep production is not known. The expansion of the blood volume and the consequent need for red blood cell production is high in the neonatal period. Also, the Ep sensitivity of the red cell progenitor, the $\mathrm{CFU}_{\mathrm{E}}$, decreases, reaching adult levels between day 8 and $20(18$, 
19). This could explain the high plasma ESF levels found around day 20 and also that ESF have been shown to increase further after hematocrit started to rise (2): However, a previous study on hypertransfused mice indicated that the high plasma ESF in 20day-old animals could not be due to the moderate concomitant postnatal anemia alone (3). In addition, Meberg et al. (2) showed that plasma ESF remained high until growth ceased between day 40 and 50, despite the hematocrit reaching adult levels beforehand. In accordance with their hypothesis, it is possible that factors related to the rapid growth could stimulate Ep production.

Acknowledgment. The authors thank Kirsten M. Østbye for excellent technical assistance.

\section{REFERENCES}

I. Krystal G 1983 Physiological and biological characterization of erythroblast cnhancing factor (EEF). a late acting erythropoietic stimulator in serum distinct from erythropoietin. Exp Hematol 11:18-31

2. Mcberg A. Hågå $\mathrm{P}$, Johansen $\mathrm{M} 1980$ Plasma erythropoietin levels in mice during the growth period. Br J Haematol 45:569-574

3. Sanengen T. Halvorsen S 1985 Regulation of erythropoiesis during rapid growth. Br J Haematol 61:273-279

4. Kreyberg L 1952 The origin and development of the "White Label" mouse strain. Br J Cancer 6:140-147

5. Widerøc T-E, Sanengen T, Halvorsen S 1983 Erythropoietin and uremic toxicity during continuous ambulatory peritoneal dialysis. Kidney Int 24(suppl 16):S208-S217

6. Hågå P. Falkanger B 1979 In vitro assay for erythropoietin: erythroid colony formation in methyl cellulose used for the measurement of erythropoietin in plasma. Blood 53:1172-1181

7. Spivak JL, Small D, Shaper JM, Hollenberg MD 1978 Use of immobilized lectins and other ligands for the partial purification of erythropoietin. Blood 52:1178-1188

8. Krystal G, Eaves AC, Eaves CJ 1981 Determination of normal human serum erythropoietin levels, using mouse bone marrow. J Lab Clin Med 97:158169

9. Congote LF 1983 Isolation of two biologically active peptides, erythrotropin I and erythrotropin II from fetal intestine. Biochem Biophys Res Commun 115:477-483

10. Congote LF 1984 Extraction from fetal bovine serum of erythrotropin, an erythroid cell-stimulating factor. Anal Biochem 140:428-433

11. Firkin FC, Russel SH 1983 Influence of human serum components on measurement of erythropoietin biological activity in vitro. Scand J Haematol 31:349-358

12. Kurtz A, Jelkman W, Bauer C 1982 A new candidate for the regulation of erythropoiesis. Insulin-like growth factor I. FEBS Lett 149:105-108

13. Kurtz A, Jelkman W, Bauer C 1983 Insulin stimulates erythroid colony formation independently of erythropoietin. Br J Haematol 53:311-316

14. Krystal G 1983 A simple microassay for erythropoietin based on ${ }^{3} \mathrm{H}$-thymidine incorporation into spleen cells from phenylhydrazine treated mice. Exp Hematol 11:649-660

15. Underwood LE, D'Ercole AJ, Van Wyk JJ 1980 Somatomedin-C and the assessment of growth. Pediatr Clin North Am 27:771-782

16. D'Ercole AJ, Applewhite GT, Underwood LE 1980 Evidence that somatomedin is synthesized by multiple tissues in the fetus. Dev Biol 75:315-328

1.7. Jelkmann W, Kurtz A, Bauer C.1983 Extraction of erythropoietin from isolated renal glomeruli of hypoxic rats. Exp Hematol 1 1:581-588

18. Rich IN. Kubanek B 1980 The ontogeny of erythropoiesis in the mouse detected by the erythroid colony-forming technique. II. Transition in erythropoietin sensitivity during development. J Embryol Exp Morphol 58:143155

19. Hall EA, Shinpock SG, Goodman JW 1984 In vitro studies of erythropoietic progenitors (CFU-E) in marrow from neonatal and young mice. Exp Hematol 12:549-557 